\title{
Osteoporose masculina idiopática - Um caso clínico
}

\author{
Idiopathic Male osteoporosis - Case report
}

\author{
Inês Vidal' ${ }^{1}$, Ana Catarina Lucas ${ }^{1}$, Pedro Ribeiro ${ }^{2}$, Adriano Rodrigues ${ }^{3}$ \\ ${ }^{\top}$ Interna da Formação Específica em Medicina Interna, Serviço de Medicina Interna, CHUC-HG, Coimbra, Portugal \\ ${ }^{2}$ Assistente Graduado em Medicina Interna, Serviço de Medicina Interna, CHUC-HG, Coimbra, Portugal \\ ${ }^{3}$ Director de Serviço, Serviço de Medicina Interna, CHUC-HG, Coimbra, Portugal
}

\section{Resumo}

Doente do sexo masculino, de 49 anos, internado no Serviço de Medicina de um Hospital Central para estudo de múltiplas fracturas de costelas de origem não traumática. Apresentava uma densitometria óssea, realizada em ambulatório, com critérios de osteoporose e uma biópsia óssea de um dos locais de fractura que revelava apenas processo cicatricial. 0 estudo etiológico realizado não permitiu identificar uma causa para a osteoporose, estabelecendo-se o diagnóstico de osteoporose masculina idiopática, e iniciando-se terapêutica anti-reabsortiva.

A osteoporose masculina idiopática normalmente ocorre em homens de meia-idade que se apresentam com fractura ou dor lombar de causa não traumática e nos quais não se encontra uma etiologia que o justifique.

Palabras clave: Osteoporosis. Idiopática. Hombre. Fractura.

\section{Introdução}

A osteoporose é uma doença silenciosa que se caracteriza por baixa massa óssea com disrupção arquitectural e fragilidade óssea, resultando num maior risco de fractura., ${ }^{1,2}$ Apesar de mais frequente no sexo feminino, os homens também são afectados. ${ }^{2,3}$ Estudos epidemiológicos mostram que a etiologia no homem pode ser identificada em 40 - 60\% dos casos, sendo os restantes designados por osteoporose idiopática. ${ }^{4-6}$

A osteoporose masculina idiopática normalmente ocorre em homens de meia-idade que se apresentam com fractura ou dor lombar de causa não traumática e sem alterações laboratoriais. ${ }^{3,4}$ Se seguirmos os critérios da OMS, a prevalência da osteoporose masculina é de cerca de $4.15 \%$. 0 custo financeiro associado à osteoporose masculina foi de quase 11,6 bilhões de euros na União Europeia. Projecções sugerem que o número total de fraturas aumentará em 34\% até 2025, atingindo quase 1,6 milhões de casos por ano, correspondendo a um custo total de cerca de 15,5 biliões de euros. ${ }^{\text {? }}$

0 presente caso clínico pretende recordar a existência desta entidade que, apesar de pouco estudada, está presente em cerca de $50 \%$ dos homens com osteoporose. É importante realizar um estudo detalhado para excluir causas identificáveis e muitas vezes reversiveis de osteoporose no homem, estabelecer o diagnóstico e iniciar terapêutica adequada de forma a limitar a sua progressão.

\section{Caso clínico}

Doente do sexo masculino, de 49 anos, internado electivamente no Serviço de Medicina de um Hospital Central para

\begin{abstract}
Male patient, 49 years old, was admitted to study multiple fractures of ribs, of nontraumatic origin. He had a bone densitometry performed on an outpatient basis with osteoporosis criteria, and a bone biopsy of the fracture site that revealed only healing process. The etiologic study not identified a cause for osteoporosis, establishing the diagnosis of idiopathic male osteoporosis and starting up anti-reabsorptive therapy.

Idiopathic male osteoporosis usually occurs in middle-aged men who present with fractures or back pain from non-traumatic causes and which is not an etiology that justifies.
\end{abstract}

Key words: Osteoporosis. Idiopathic. Man. Fracture

estudo de múltiplas fracturas de costelas de etiologia não traumática. Trata-se de um indivíduo previamente saudável, sem patologias conhecidas, sem medicação crónica e sem antecedentes familiares de relevo. Negava hábitos tabágicos e etilicos.

0 doente apresentava uma densitometria óssea, realizada em ambulatório, com critérios de osteoporose (Score T = -2.8) e uma biópsia óssea de um dos locais de fractura que revelava apenas um processo cicatricial.

No internamento, foi realizado estudo etiológico abrangente (Tabela 1), de forma a excluir causas secundárias de osteo-

Tabela 1. Exames analíticos para estudo etiológico de osteoporose no homem

\begin{tabular}{|l|l|}
\hline \multicolumn{1}{|c|}{ Exames básicos } & \multicolumn{1}{|c|}{$\begin{array}{c}\text { Exames específicos em casos } \\
\text { particulares }\end{array}$} \\
\hline Hemograma com leucograma & $\begin{array}{l}\text { Marcadores de formação e } \\
\text { reabsorção óssea }\end{array}$ \\
\hline Função renal & $\begin{array}{l}\text { Hormonas - testosterona, } \\
\text { estradiol, prolactina, progesterona, } \\
\text { PTH, cortisol, osteocalcina, IGF-1, } \\
\text { função tiroideia }\end{array}$ \\
\hline lonograma & Cortisol livre na urina de 24h \\
\hline Fosfatase alcalina & Triptase sérica \\
\hline Função hepática & HbA1c \\
\hline $\begin{array}{l}\text { Electroforese das proteínas } \\
\text { séricas e urinárias }\end{array}$ & 25-hidroxivitamina-D \\
\hline Cálcio na urina das 24h & SACE \\
\hline $\begin{array}{l}\text { Estudo auto-imune - Ac anti- } \\
\text { transglutaminase, para excluir } \\
\text { doença celíaca, Ac anti-tiroideus }\end{array}$ & \\
\hline
\end{tabular}


porose. Realizou hemograma, velocidade de sedimentação, função renal, ionograma com cálcio sérico, e fosfatase alcalina, apresentando todos os valores dentro dos parâmetros da normalidade. Realizou ainda electroforese das proteínas séricas que não revelou alterações, estudo auto-imune (incluindo SACE, Ac anti-tiroideus, Ac anti-TPO, Ac anti-TG) que foi negativo, e estudo hormonal, com doseamento de estradiol, prolactina, progesterona, testosterona total, cortisol, PTH, osteocalcina, IGF-I, TSH, T3 e T4 livres, com todos os valores dentro do intervalo de normalidade. Completouse 0 estudo com determinação da hemoglobina glicosilada que era de $5.4 \%$ e determinação dos marcadores tumorais (CEA, CA 19.9, PSA, CYFRA, Neuroenolase específica, alfafetoproteína e beta2 microglobulina) que foram negativos. Estabeleceu-se então o diagnóstico de osteoporose masculina idiopática, um diagnóstico de exclusão.

Como terapêutica, fez toma única de pamidronato e iniciou calcitriol durante 0 internamento. À data da alta, foi recomendada uma alimentação rica em cálcio e foi medicado com alendronato semanal.

Após 3 anos com esta terapêutica não voltou a apresentar fracturas.

\section{Discussão}

No homem, tal como na mulher, a incidência de fracturas osteoporóticas aumenta com a idade, mas começa, comparativamente, cerca de dez anos mais tarde. ${ }^{4,1-3}$

A densidade mineral óssea nos homens aumenta de forma marcada durante a puberdade em resposta à produção aumentada de esteróides sexuais. ${ }^{3-5} \mathrm{~A}$ idade com que se atinge a puberdade e situações patológicas como 0 hipogonadismo hipogonadotrófico são questões relevantes para determinar o risco de osteoporose..$^{1,3}$

Em termos hormonais, tanto os esteróides sexuais, como a PTH e o factor de crescimento insulina-like (IGF) são importantes para o estabelecimento e manutenção da massa óssea. ${ }^{1}$ Estudos concluiram que 0 estrogénio é 0 esteróide sexual major na regulação da reabsorção óssea, enquanto tanto o estrogénio como a testosterona são importantes para a formação óssea. ${ }^{1,4}$

A OMS define osteoporose como score $T<-2.5$ em homens com mais de 65 anos. Entre os 50 e os 65 anos, para além de um score $T<-2.5$ será necessário existir um ou mais factores de risco para fractura para se estabelecer o diagnóstico de osteoporose. 2,4

A prevalência de osteoporose no sexo masculino é de 4-6\% acima dos 50 anos, com 33-47\% dos homens a preencher critérios de osteopenia. ${ }^{2} 30 \%$ das fracturas da anca ocorrem em homens e têm duas vezes maior mortalidade que as mulheres, durante o primeiro ano. , $^{3,5}$ Não existem manifestações da doença até haver fractura e cerca de $80 \%$ das fracturas são assintomáticas. 0 diagnóstico no homem é feito quase sempre durante avaliação para estudo de fractura não traumática, sendo pouco frequente no homem a medição da densidade óssea por rotina. ${ }^{3,4} \mathrm{~A}$ osteoporose pode dever-se directamente à fisiopatologia da doença, como no hipertiroidismo, hiperparatiroidismo primário ou na doença de Cushing, pelo efeito deletério no osso do excesso de hormona tiroideia, PTH e glicocorticóides, respectivamente. Pode também ser um epifenómeno ligado indirectamente à patologia, como na doença neurológica que requer imobilização ou nas síndromes malabsortivas com consequente hiperparatiroidismo secundário. Pode ainda resultar do tratamento de certas patologias, como no caso da osteoporose induzida por glicocorticóides ou no tratamento do cancro da próstata com análogos da hormona libertadora de gonadotrofina (GnRH). Na Tabela 2 estão listadas as causas secundárias de osteoporose masculina. ${ }^{4,1,3,6}$

Cerca de 2-3\% dos homens com osteoporose masculina idiopática têm história de puberdade tardia, 0 que pode ser um percursor desta entidade. 0 défice de estrogénios tam-

Tabela 2. Causas secundárias de osteoporose

\begin{tabular}{|c|}
\hline Medicação com glicocorticóides \\
\hline $\begin{array}{l}\text { Doenças endócrinas } \\
\text { - Hipogonadismo }(+++) \\
\text { - Síndrome de Cushing } \\
\text { - Hiperparatiroidismo } \\
\text { - DM tipo } 1\end{array}$ \\
\hline $\begin{array}{l}\text { Outros medicamentos: } \\
\text { - Imunossupressores }(+++) \\
\text { - Anticonvulsivantes } \\
\text { - ISRS } \\
\text { - Glitazonas } \\
\text { - Análogos GnRH } \\
\text { - Heparina }\end{array}$ \\
\hline Abuso álccol \\
\hline Tabagismo \\
\hline $\begin{array}{l}\text { Doenças Gastrointestinais } \\
\text { - Pós- gastrectomia } \\
\text { - Doença celíaca } \\
\text { - Pós-cirurgia bariátrica } \\
\text { - Síndromes malabsortivos } \\
\text { - Doença inflamatória intestinal } \\
\text { - Cirrose Biliar primária }\end{array}$ \\
\hline DPOC e asma \\
\hline Artrite Reumatóide \\
\hline Anemia perniciosa \\
\hline Hiperhomocisteinémia \\
\hline Doenças neoplásicas \\
\hline Fibrose quística \\
\hline Imobilização \\
\hline Insuficiência renal \\
\hline Homocisteinúria \\
\hline Hemocromatose \\
\hline Hipercalciúria \\
\hline Mastocitose sistémica \\
\hline
\end{tabular}


bém pode ser responsável por alguns casos de osteoporose não explicada em alguns homens. ${ }^{4,6}$

A avaliação clínica, densitométrica, analítica e radiográfica na osteoporose masculina tem como objectivo excluir diagnósticos diferenciais possiveis, excluir causas de osteoporose secundária que possam exigir terapêuticas específicas, e estimar o risco de fractura - um elemento fundamental no processo de decisão terapêutica. ${ }^{3}$

0 estudo da densidade óssea só deverá ser realizado se houver manifestações clínicas de baixa massa óssea, como no caso de existir um RX sugestivo de osteopenia, fracturas espontâneas, perda de altura ou factores de risco para fractura. ${ }^{3,4} 0$ estudo laboratorial pode contribuir para 0 diagnóstico diferencial com mieloma múltiplo e osteomalacia, bem como para excluir causas secundárias de osteoporose. $\mathrm{Na}$ Tabela 1 estão listados os exames analíticos essenciais para estudo etiológico de osteoporose no homem. ${ }^{4}$ Raramente é necessária a realização de biópsia óssea. ${ }^{3}$

Uma das questões fundamentais na osteoporose masculina é 0 facto de apenas uma pequena percentagem dos homens com osteoporose receber tratamento específico $-4.5 \%$ VS $27 \%$ das mulheres. ${ }^{3,4}$ É por isso fundamental estar alerta que esta patologia existe também no sexo masculino, estando até associada a uma taxa de mortalidade mais elevada, para estabelecer o diagnóstico precocemente, de forma a reverter a sua progressão. 0 tratamento a aplicar deverá ser ponderado caso a caso, mas é semelhante ao utilizado no sexo feminino. Devem ser recomendadas medidas gerais como prática de exercício físico, exposição solar moderada, dieta equilibrada, suplementação com cálcio oral (1200 a $1500 \mathrm{mg}$ ) e eliminar 0 álcool e 0 tabaco. 0 tratamento farmacológico visa aumentar a formação óssea, como a PTH ou o ranelato de estrôncio, ou reduzir a reabsorção óssea, como a calcitonina, raloxifeno, testosterona, associação de cálcio com vitamina D ou os bifosfonatos.,
Tabela 3. Exames analíticos para estudo etiológico de osteoporose no homem

\begin{tabular}{|c|c|}
\hline Exames básicos & $\begin{array}{c}\text { Exames específicos em casos } \\
\text { particulares }\end{array}$ \\
\hline Hemograma com leucograma & $\begin{array}{c}\text { Marcadores de formação e } \\
\text { reabsorção óssea }\end{array}$ \\
\hline Função renal & $\begin{array}{c}\text { Hormonas - testosterona, } \\
\text { estradiol, prolactina, progesterona, } \\
\text { PTH, cortisol, osteocalcina, IGF-1, } \\
\text { função tiroideia }\end{array}$ \\
\hline Ionograma & Cortisol livre na urina de 24h \\
\hline Fosfatase alcalina & Triptase sérica \\
\hline Função hepática & HbA1c \\
\hline $\begin{array}{c}\text { Electroforese das proteínas } \\
\text { séricas e urinárias }\end{array}$ & 25-hidroxivitamina-D \\
\hline Cálcio na urina das 24h & SACE \\
\hline $\begin{array}{c}\text { Estudo auto-imune }- \text { Ac anti- } \\
\text { transglutaminase, para excluir } \\
\text { doença celíaca, Ac anti-tiroideus }\end{array}$ & \\
\hline
\end{tabular}

\section{Referências}

1. Ostertag, A., et al., A case-control study of fractures in men with idiopathic osteoporosis: Fractures are associated with older age and low cortical bone density. Bone, 2013. 52(1): p. 48-55.

2. Gielen, E., et al., Osteoporosis in men. Best Practice \& Research Clinical Endocrinology \& Metabolism, 2011. 25(2): p. 321-335.

3. Kaufman, J.M. and S. Goemaere, Osteoporosis in men. Best Practice \& Research Clinical Endocrinology \& Metabolism, 2008. 22(5): p. 787-812.

4. Luigi Gennari, J.P.B., Idiopathic Osteoporosis in Men. Curr Osteoposos Rep, 2013. 11: p. 13.

5. Herrera, A., Male osteoporosis: A review. World Orthop, 2012. 3(12): p. 12.

6. Laroche, M., Pattern of bone mineral density in idiopathic male osteoporosis. Rheumatology International, 2012. 32(10): p. 3093-3096.

7. International Osteoporosis Foundation, disponível em http://share.iofbonehealth.org/ WOD/2014/thematic-report/WOD14-Report-PT_BR.pdf 